Article

\title{
Existence, Uniqueness and Ulam's Stability of Solutions for a Coupled System of Fractional Differential Equations with Integral Boundary Conditions
}

\author{
Dimplekumar Chalishajar ${ }^{1, *}$ and Avadhesh Kumar ${ }^{2}$ \\ 1 Department of Mathematics and Computer Science, Mallory Hall, Virginia Military Institute, \\ Lexington, VA 24450, USA \\ 2 School of Basic Sciences, Indian Institute of Technology Mandi, Kamand 175 005, H.P., India; \\ soni.iitkgp@gmail.com \\ * Correspondence: chalishajardn@vmi.edu
}

Received: 2 May 2018; Accepted: 29 May 2018; Published: 7 June 2018

\begin{abstract}
In this paper, the existence and uniqueness of the solutions to a fractional order nonlinear coupled system with integral boundary conditions is investigated. Furthermore, Ulam's type stability of the proposed coupled system is studied. Banach's fixed point theorem is used to obtain the existence and uniqueness of the solutions. Finally, an example is provided to illustrate the analytical findings.
\end{abstract}

Keywords: coupled system; green's function; integral boundary conditions; Ulam's stability

AMS Subject Classification: 34A08; 34B15; 34B27; 35B35

\section{Introduction}

Fractional calculus is a branch of mathematical analysis, in which arbitrary order differential and integral operators are studied. It started with a correspondence between L'Hospital and Leibnitz in 1695. Presently, plenty of literature is available on theoretical as well as numerical work on this topic. It has application in numerous fields, for example, control theory, signal and image processing, aerodynamics and biophysics [1-3]. For the fundamental concepts of fractional calculus, books like Kilbas et al. [4], Miller and Ross [5] and Halfer [6] are referred. Existence and uniqueness of solutions for fractional order differential systems in finite dimensional as well infinite dimensional spaces were studied by several authors [7-11]. Ahmad et al. [8] established existence results for nonlinear boundary value fractional integro-differential equations with integral boundary conditions.

Integral boundary conditions have several applications in real-life problems such as population dynamics, blood flow problems, underground water flow, and chemical engineering. For more details on integral boundary conditions, we refer the reader to [12]. Here we would like to consider a practical example of the integral boundary condition:

$$
-x^{\prime \prime}=g(t) f(t, x), x(0)=0, \beta x^{\prime}(1)=x(\eta),
$$

where $t \in(0,1), \eta \in(0,1]$ and $\beta$ is a positive constant. This is the model for a thermostat. Solutions of the problem are stationary solutions for a one-dimensional heat equation, corresponding to a heated bar, with a controller at 1 , which adds or removes heat, depending on the temperature detected by a sensor at $\eta$.

This problem can be generalized. One can consider the heat equation with nonlinear gradient source terms that vary in time. Moreover, now, the heated bar, with a controller at 1, which adds 
or removes heat depending on the temperature detected by sensors located at any points of the bar (it depends on how we define the function $h$ ). This problem can be written in the form

$$
x^{\prime \prime}=f\left(t, x, x^{\prime}\right), x(0)=0, x^{\prime}(1)=\int_{0}^{1} x(s) d h(s)(\eta)
$$

Recently, Ulam's type stability has been of great interest to many researchers. In 1940, the above mentioned stability was first introduced by Ulam [13]. Then, it was explained by Hyers [14] in the subsequent years. Ulam and Hyers studied it for various kinds of differential equations with integer order. Nowadays, we describe the result of Hyers simply saying that Cauchy functional equation is Hyers-Ulam stable (or has the Hyers-Ulam stability). Next, Hyers and Ulam published some further stability results for polynomial functions, isometries, and convex functions. The Hyers' results are extended and generalized by many researchers for integer order differential equations. Plenty of significant results on Ulam's type stability can be found in the literature, we refer [15-17] and references cited therein.

To the best of our knowledge, there are only few manuscripts devoted to the study of Ulam's type stability for coupled system of fractional differential equations. Further, there is no manuscript considering the Ulam's stability for coupled system of fractional order $\alpha \in(1,2]$ differential equations with integral boundary conditions. Motivated by this fact, in this paper, the existence, uniqueness of solutions as well as Ulam's type stability for the considered coupled system involving Caputo derivative is studied. The proposed system is given as follows:

$$
\left\{\begin{array}{l}
{ }^{c} D^{\alpha} x(t)=f(t, y(t)), \alpha \in(1,2], t \in \mathcal{J} \\
{ }^{c} D^{\beta} y(t)=g(t, x(t)), \beta \in(1,2], t \in \mathcal{J} \\
p x(0)+q x^{\prime}(0)=\int_{0}^{1} a_{1}(x(s)) d s, \quad p x(1)+q x^{\prime}(1)=\int_{0}^{1} a_{2}(x(s)) d s, \\
\tilde{p} y(0)+\tilde{q} y^{\prime}(0)=\int_{0}^{1} \tilde{a}_{1}(y(s)) d s, \quad \tilde{p} y(1)+\tilde{q} y^{\prime}(1)=\int_{0}^{1} \tilde{a}_{2}(y(s)) d s,
\end{array}\right.
$$

where $\mathcal{J}=[0,1]$ and $f, g: \mathcal{J} \times \mathbb{R} \rightarrow \mathbb{R}$ are continuous functions. Here, $a_{1}, a_{2}, \tilde{a}_{1}, \tilde{a}_{2}: \mathbb{R} \rightarrow \mathbb{R}$ and $p, \tilde{p}>0 ; q, \tilde{q} \geq 0$ are real numbers.

The plan of the paper is as follows. In the second section, some useful definitions, notations, lemmas and results are given which will be required for the later sections. In the third section, existence and uniqueness of the solutions for the coupled system (1) is studied. In the fourth section, Ulam's type stability results are obtained. In the last section, a few examples are given to show the application of the obtained abstract results.

\section{Preliminaries and Assumptions}

In this section, some useful definitions, notations and lemmas are briefly reviewed.

Definition 1. [4] For any function $z \in((0,1), \mathbb{R})$, the Caputo derivative of fractional order $\alpha \in \mathbb{R}^{+}$is defined as

$$
{ }^{c} D^{\alpha} z(t)=\frac{1}{\Gamma(n-\alpha)} \int_{0}^{t}(t-s)^{n-\alpha-1} z^{(n)}(s) d s, \quad n=[\alpha]+1,
$$

where $[\alpha]$ denotes the integer part of $\alpha$ and $\Gamma(\cdot)$ is the gamma function.

Definition 2. [4] The Riemann-Liouville fractional integral of order $\alpha \in \mathbb{R}^{+}$is defined by

$$
J^{\alpha} z(t)=\frac{1}{\Gamma(\alpha)} \int_{0}^{t}(t-s)^{\alpha-1} z(s) d s
$$


where $z(t) \in L^{1}\left([0,1], \mathbb{R}^{+}\right)$.

Lemma 1. [4] For any $\alpha>0$, then the differential equations

$$
{ }^{c} D^{\alpha} z(t)=0
$$

has solution given by

$$
z(t)=c_{0}+c_{1} t+c_{2} t^{2}+\cdots+c_{n-1} t^{n-1}, \quad c_{i} \in \mathbb{R}, i=0,1, \cdots, n-1,
$$

where $n=[\alpha]+1$.

Lemma 2. [4] For any $\alpha>0$, then the solution of the differential equations

$$
{ }^{c} D^{\alpha} z(t)=h(t)
$$

will be given by

$$
J^{\alpha}\left[{ }^{c} D^{\alpha} z(t)\right]=J^{\alpha} z(t)+c_{0}+c_{1} t+c_{2} t^{2}+\cdots+c_{n-1} t^{n-1}, \quad c_{i} \in \mathbb{R}, i=0,1, \cdots, n-1,
$$

where $n=[\alpha]+1$.

Lemma 3. [8] For any $h, \gamma_{1}, \gamma_{2} \in C([0,1], \mathbb{R})$, the unique solution of the boundary value problem

$$
\left\{\begin{array}{l}
{ }^{c} D^{\alpha} z(t)=h(t), \alpha \in(1,2], t \in[0,1] \\
p z(0)+q z^{\prime}(0)=\int_{0}^{1} \gamma_{1}(s) d s, \quad p z(1)+q z^{\prime}(1)=\int_{0}^{1} \gamma_{2}(s) d s,
\end{array}\right.
$$

is given by

$$
z(t)=\int_{0}^{1} G_{\alpha}(t, s) h(s) d s+\frac{1}{p^{2}}\left[(p(1-t)+q) \int_{0}^{1} \gamma_{1}(s) d s+(q+p t) \int_{0}^{1} \gamma_{2}(s) d s\right],
$$

where $G_{\alpha}(t, s)$ is the Green's function given by

$$
G_{\alpha}(t, s)=\left\{\begin{array}{l}
\frac{p(t-s)^{\alpha-1}+(q-p t)(1-s)^{\alpha-1}}{p \Gamma(\alpha)}+\frac{q(q-p t)(1-s)^{\alpha-2}}{p^{2} \Gamma(\alpha-1)}, s \leq t, \\
\frac{(q-p t)(1-s)^{\alpha-1}}{p \Gamma(\alpha)}+\frac{q(q-p t)(1-s)^{\alpha-2}}{p^{2} \Gamma(\alpha-1)}, t \leq s .
\end{array}\right.
$$

Lemma 4. The space $\mathcal{B}=\{z(t) \mid z \in C(\mathcal{J})\}$ is a Banach space under the defined norm $\|z\|_{\mathcal{B}}=$ $\max _{t \in \mathcal{J}}|z(t)|$. Similarly, the norm on product space is defined by $\|(z, \tilde{z})\|_{\mathcal{B} \times \mathcal{B}}=\|z\|_{\mathcal{B}}+\|\tilde{z}\|_{\mathcal{B}}$. Obviously $\left(\mathcal{B} \times \mathcal{B},\|(\cdot, \cdot)\|_{\mathcal{B} \times \mathcal{B}}\right)$ is a Banach space. Further, the cone $\mathcal{C} \subset \mathcal{B} \times \mathcal{B}$ is defined by

$$
\mathcal{C}=\{(z, \tilde{z}) \in \mathcal{B} \times \mathcal{B} \mid z(t) \geq 0, \tilde{z}(t) \geq 0\} .
$$

Here, the problem (1) is transformed into a fixed point problem. Let $\mathcal{F}: \mathcal{B} \times \mathcal{B} \rightarrow \mathcal{B} \times \mathcal{B}$ be the operator defined as 


$$
\begin{aligned}
\mathcal{F}(x, y)(t)= & \left(\begin{array}{c}
\int_{0}^{1} G_{\alpha}(t, s) f(s, y(s)) d s+\frac{1}{p^{2}}\left[(p(1-t)+q) \int_{0}^{1} a_{1}(x(s)) d s\right. \\
\left.+(q+p t) \int_{0}^{1} a_{2}(x(s)) d s\right] \\
\int_{0}^{1} G_{\beta}(t, s) g(s, x(s)) d s+\frac{1}{\tilde{p}^{2}}\left[(\tilde{p}(1-t)+\tilde{q}) \int_{0}^{1} \tilde{a}_{1}(y(s)) d s\right. \\
\left.+(\tilde{q}+\tilde{p} t) \int_{0}^{1} \tilde{a}_{2}(y(s)) d s\right]
\end{array}\right) \\
= & \left(\begin{array}{c}
\mathcal{F}_{\alpha}(y, x)(t) \\
\mathcal{F}_{\beta}(x, y)(t)
\end{array}\right) .
\end{aligned}
$$

Then the fixed point of the operator $\mathcal{F}$ coincides with the solution of coupled system (1).

In order to prove the existence and uniqueness of solutions of coupled system (1), following assumptions are taken:

(A1) For $t \in \mathcal{J}$, there exist $\lambda, \mu \in C\left(\mathcal{J}, \mathbb{R}^{+}\right)$, such that

$$
|f(t, y(t))| \leq \lambda(t)+\mu(t)|y(t)|, \forall y(t) \in C(\mathcal{J}, \mathbb{R})
$$

with $\lambda^{*}=\sup _{t \in \mathcal{J}} \lambda(t), \mu^{*}=\sup _{t \in \mathcal{J}} \mu(t)$.

Similarly, for $t \in \mathcal{J}$, there exist $v, \xi \in C\left(\mathcal{J}, \mathbb{R}^{+}\right)$, such that

$$
|g(t, x(t))| \leq v(t)+\xi(t)|x(t)|, \forall x(t) \in C(\mathcal{J}, \mathbb{R})
$$

with $v^{*}=\sup _{t \in \mathcal{J}} v(t), \xi^{*}=\sup _{t \in \mathcal{J}} \xi(t)$.

(A2) For $t \in \mathcal{J}$, there exist positive constant $L_{a_{1}}, L_{a_{2}}$, such that

$$
\left|a_{1}(x(t))\right| \leq L_{a_{1}}|x(t)| \text { and }\left|a_{2}(x(t))\right| \leq L_{a_{2}}|x(t)|, \forall x(t) \in C(\mathcal{J}, \mathbb{R})
$$

Similarly, For $t \in \mathcal{J}$, there exist positive constant $L_{\tilde{a}_{1}}, L_{\tilde{a}_{2}}$, such that

$$
\left|\tilde{a}_{1}(y(t))\right| \leq L_{\tilde{a}_{1}}|y(t)| \text { and }\left|\tilde{a}_{2}(x(t))\right| \leq L_{\tilde{a}_{2}}|y(t)|, \forall y(t) \in C(\mathcal{J}, \mathbb{R})
$$

(A3)

$$
\mathbb{P}_{1}=\int_{0}^{1}\left|G_{\alpha}(t, s)\right| \lambda(s) d s<\infty, \mathbb{Q}_{1}=\int_{0}^{1}\left|G_{\alpha}(t, s)\right| \mu(s) d s+\frac{(p+q)\left(L_{a_{1}}+L_{a_{2}}\right)}{p^{2}}<\frac{1}{2}
$$

and

$$
\mathbb{P}_{2}=\int_{0}^{1}\left|G_{\beta}(t, s)\right| v(s) d s<\infty, \mathbb{Q}_{2}=\int_{0}^{1}\left|G_{\beta}(t, s)\right| \xi(s) d s+\frac{(\tilde{p}+\tilde{q})\left(L_{\tilde{a}_{1}}+L_{\tilde{a}_{2}}\right)}{\tilde{p}^{2}}<\frac{1}{2} .
$$

(A4) For all $y, \tilde{y} \in C(\mathcal{J}, R)$ and for each $t \in \mathcal{J}$ there exists a positive constant $K_{f}$, such that

$$
|f(t, y)-f(t, \tilde{y})| \leq K_{f}|y-\tilde{y}| .
$$

Similarly, for all $x, \tilde{x} \in C(\mathcal{J}, R)$ and for each $t \in \mathcal{J}$ there exists a positive constant $K_{g}$, such that

$$
|g(t, x)-g(t, \tilde{x})| \leq K_{g}|x-\tilde{x}| .
$$

(A5) For all $x, \tilde{x} \in C(\mathcal{J}, R)$ and for each $t \in \mathcal{J}$ there exist positive constants $K_{a_{1}}, K_{a_{2}}$, such that

$$
\left|a_{1}(x)-a_{1}(\tilde{x})\right| \leq K_{a_{1}}|x-\tilde{x}| \text { and }\left|a_{2}(x)-a_{2}(\tilde{x})\right| \leq K_{a_{2}}|x-\tilde{x}| .
$$


Similarly, for all $y, \tilde{y} \in C(\mathcal{J}, R)$ and for each $t \in \mathcal{J}$ there exist positive constants $K_{\tilde{a}_{1}}, K_{\tilde{a}_{2}}$, such that

$$
\left|\tilde{a}_{1}(y)-\tilde{a}_{1}(\tilde{y})\right| \leq K_{\tilde{a}_{1}}|y-\tilde{y}| \text { and }\left|\tilde{a}_{2}(y)-\tilde{a}_{2}(\tilde{y})\right| \leq K_{\tilde{a}_{2}}|y-\tilde{y}| .
$$

(A6) Let

(i) $\phi_{1}=\mathcal{K}_{(\beta \tilde{p} \tilde{q})} K_{g}+K_{p q}$, where $K_{p q}=\frac{(p+q)\left(K_{a_{1}}+K_{a_{2}}\right)}{p^{2}}$ and

$$
\begin{aligned}
\mathcal{K}_{(\beta \tilde{p} \tilde{q})} & =\max _{t \in[0,1]}\left|\int_{0}^{1} G_{\beta}(t, s) d s\right| \\
& =\left|\int_{0}^{t}\left[\frac{\tilde{p}(t-s)^{\beta-1}+(\tilde{q}-\tilde{p} t)(1-s)^{\beta-1}}{\tilde{p} \Gamma(\beta)}+\frac{\tilde{q}(\tilde{q}-\tilde{p} t)(1-s)^{\beta-2}}{\tilde{p}^{2} \Gamma(\beta-1)}\right] d s\right| \\
& +\left|\int_{t}^{1}\left[\frac{(\tilde{q}-\tilde{p} t)(1-s)^{\beta-1}}{\tilde{p} \Gamma(\beta)}+\frac{\tilde{q}(\tilde{q}-\tilde{p} t)(1-s)^{\beta-2}}{\tilde{p}^{2} \Gamma(\beta-1)}\right] d s\right| \\
& =\frac{1}{\Gamma(\beta+1)}+\frac{2(\tilde{p}+\tilde{q})}{\Gamma(\beta+1)}+\frac{2\left(\tilde{q}^{2}+\tilde{p} \tilde{q}\right)}{\tilde{p}^{2} \Gamma(\beta)}
\end{aligned}
$$

(ii) $\phi_{2}=\mathcal{K}_{(\alpha p q)} K_{f}+K_{\tilde{p} \tilde{q}}$, where $K_{\tilde{p} \tilde{q}}=\frac{(\tilde{p}+\tilde{q})\left(K_{\tilde{a}_{1}}+K_{\tilde{a}_{2}}\right)}{\tilde{p}^{2}}$ and

$$
\begin{aligned}
\mathcal{K}_{(\alpha p q)} & =\max _{t \in[0,1]}\left|\int_{0}^{1} G_{\alpha}(t, s) d s\right| \\
& =\frac{1}{\Gamma(\alpha+1)}+\frac{2(p+q)}{\Gamma(\alpha+1)}+\frac{2\left(q^{2}+p q\right)}{p^{2} \Gamma(\alpha)} .
\end{aligned}
$$

\section{Existence and Uniqueness Analysis}

Theorem 1. If all the assumptions (A1)-(A6) and $\phi=\max \left\{\phi_{1}, \phi_{2}\right\}<1$ are fulfilled, then the fractional order coupled system (1) has a unique solution.

Proof. For a positive number

$$
\delta=\max \left(\frac{2 \mathbb{P}_{1}}{1-2 \mathbb{Q}_{1}}, \frac{2 \mathbb{P}_{2}}{1-2 \mathbb{Q}_{2}}\right)
$$

we define a set

$$
\mathcal{W}=\left\{(x, y) \in \mathcal{B} \times \mathcal{B}:\|(x, y)\|_{\mathcal{B} \times \mathcal{B}} \leq \delta\right\} .
$$

First, in order to prove that $\mathcal{F}$ maps $\mathcal{W}$ into itself, we have

$$
\begin{aligned}
\left|\mathcal{F}_{\alpha}(y, x)(t)\right| \leq & \int_{0}^{1}\left|G_{\alpha}(t, s)\right||f(s, y(s))| d s \\
& +\frac{1}{p^{2}}\left[|(p(1-t)+q)| \int_{0}^{1}\left|a_{1}(x(s))\right| d s+|(q+p t)| \int_{0}^{1}\left|a_{2}(x(s))\right| d s\right] \\
\leq & \int_{0}^{1}\left|G_{\alpha}(t, s)\right| \lambda(s) d s+\int_{0}^{1}\left|G_{\alpha}(t, s)\right|[\mu(s)|y(s)|] d s \\
& +\frac{1}{p^{2}}\left[(p+q) \int_{0}^{1}\left|a_{1}(x(s))\right| d s+(p+q) \int_{0}^{1}\left|a_{2}(x(s))\right| d s\right] \\
\leq & \int_{0}^{1}\left|G_{\alpha}(t, s)\right| \lambda(s) d s+\delta\left[\int_{0}^{1}\left|G_{\alpha}(t, s)\right| \mu(s) d s+\frac{(p+q)\left(L_{a_{1}}+L_{a_{2}}\right)}{p^{2}}\right] \\
& =\mathbb{P}_{1}+\delta \mathbb{Q}_{1} \leq \frac{\delta}{2} .
\end{aligned}
$$


Now taking maximum on both side of the inequality (6) over $\mathcal{J}$, we obtain

$$
\left\|\mathcal{F}_{\alpha}(y, x)\right\|_{\mathcal{B}} \leq \frac{\delta}{2}
$$

Similarly, $\left\|\mathcal{F}_{\beta}(x, y)\right\|_{\mathcal{B}} \leq \frac{\delta}{2}$. Hence, we can conclude that

$$
\|\mathcal{F}(x, y)\|_{\mathcal{B} \times \mathcal{B}} \leq \delta
$$

Inequality (7) shows that $\mathcal{F}$ maps $\mathcal{W}$ into itself. Next, in order to show that $\mathcal{F}$ is the contraction operator when $t \in \mathcal{J}$, we have

$$
\begin{aligned}
\left|\mathcal{F}_{\alpha}(y, x)(t)-\mathcal{F}_{\alpha}(\bar{y}, \bar{x})(t)\right| \leq & \int_{0}^{1}\left|G_{\alpha}(t, s)\right||f(s, y(s))-f(s, \bar{y}(s))| d s \\
& +\frac{1}{p^{2}}\left[|(p(1-t)+q)| \int_{0}^{1}\left|a_{1}(x(s))-a_{1}(\bar{x}(s))\right| d s\right. \\
& \left.+|(q+p t)| \int_{0}^{1}\left|a_{2}(x(s))-a_{2}(\bar{x}(s))\right| d s\right] \\
\leq & \mathcal{K}_{(\alpha p q)} K_{f}|y(t)-\bar{y}(t)| \\
& +\frac{(p+q)\left(K_{a_{1}}+K_{a_{2}}\right)}{p^{2}}|x(t)-\bar{x}(t)| .
\end{aligned}
$$

When we take maximum on both side of the inequality (8) over $\mathcal{J}$, we obtain

$$
\left\|\mathcal{F}_{\alpha}(y, x)-\mathcal{F}_{\alpha}(\bar{y}, \bar{x})\right\|_{\mathcal{B}} \leq \mathcal{K}_{(\alpha p q)} K_{f}\|y-\bar{y}\|_{\mathcal{B}}+\frac{(p+q)\left(K_{a_{1}}+K_{a_{2}}\right)}{p^{2}}\|x-\bar{x}\|_{\mathcal{B}}
$$

Similarly, the following can be obtained

$$
\left\|\mathcal{F}_{\beta}(x, y)-\mathcal{F}_{\beta}(\bar{x}, \bar{y})\right\|_{\mathcal{B}} \leq \mathcal{K}_{(\beta \tilde{q} \tilde{q})} K_{g}\|x-\bar{x}\|_{\mathcal{B}}+\frac{(\tilde{p}+\tilde{q})\left(K_{\tilde{a}_{1}}+K_{\tilde{a}_{2}}\right)}{\tilde{p}^{2}}\|y-\bar{y}\|_{\mathcal{B}}
$$

From (9) and (9), we get

$$
\|\mathcal{F}(x, y)-\mathcal{F}(\bar{x}, \bar{y})\|_{\mathcal{B} \times \mathcal{B}} \leq \phi\|(x, y)-(\bar{x}, \bar{y})\|_{\mathcal{B} \times \mathcal{B}} .
$$

Thus, the operator $\mathcal{F}$ is strict contraction. By Banach's fixed point method, it has a unique fixed point which is the unique solution of the considered coupled system (1).

\section{Ulam's Stability Analysis}

In this section, we study Ulam's type stability for the coupled system (1).

For some $\epsilon=\left(\epsilon_{\alpha}, \epsilon_{\beta}\right)>0$, we consider the following inequality

$$
\left\{\begin{array}{l}
\left|{ }^{c} D^{\alpha} x(t)-f(t, y(t))\right| \leq \epsilon_{\alpha}, \quad t \in \mathcal{J}, \\
\left|{ }^{c} D^{\beta} y(t)-g(t, x(t))\right| \leq \epsilon_{\beta}, \quad t \in \mathcal{J} .
\end{array}\right.
$$

The following definitions are inspired by Rus [18].

Definition 3. The coupled system (1) is said to be Ulam-Hyers stable, if there exist $\mathcal{K}_{(\alpha \beta p q \tilde{p} \tilde{q})}=$ $\left(\mathcal{K}_{(\alpha p q)}, \mathcal{K}_{(\beta \tilde{p} \tilde{q})}\right)>0$ such that for every solution $(x, y) \in \mathcal{B} \times \mathcal{B}$ of the inequality (12), there exists a unique solution $(\vartheta, \kappa) \in \mathcal{B} \times \mathcal{B}$ with

$$
|(x, y)(t)-(\vartheta, \kappa)(t)| \leq \mathcal{K}_{(\alpha \beta p q \tilde{q} \tilde{q})} \epsilon, \quad t \in \mathcal{J} .
$$


Definition 4. The coupled system (1) is said to be generalized Ulam-Hyers stable, if there exist $\Psi \in C\left(\mathbb{R}^{+}, \mathbb{R}^{+}\right)$ with $\Psi(0)=0$, such that for every solution $(x, y) \in \mathcal{B} \times \mathcal{B}$ of the inequality (12), there exist a unique solution $(\vartheta, \kappa) \in \mathcal{B} \times \mathcal{B}$ of the system (1) which satisfies

$$
|(x, y)(t)-(\vartheta, \kappa)(t)| \leq \Psi(\epsilon), \quad t \in \mathcal{J}
$$

Remark 1. Let $(x, y) \in \mathcal{B} \times \mathcal{B}$ is a solution of the system of inequality (12) if there exist functions $\phi, \psi \in C(\mathcal{J}, \mathbb{R})$ which depend upon $x$ and $y$ respectively, such that

(R1) $|\phi(t)| \leq \epsilon_{\alpha},|\psi(t)| \leq \epsilon_{\beta}, t \in \mathcal{J}$;

(R2) and

$$
\left\{\begin{array}{l}
{ }^{c} D^{\alpha} x(t)=f(t, y(t))+\phi(t), \quad t \in \mathcal{J} \\
{ }^{c} D^{\beta} y(t)=g(t, x(t))+\psi(t), \quad t \in \mathcal{J} .
\end{array}\right.
$$

Lemma 5. Let $(x, y) \in \mathcal{B} \times \mathcal{B}$ be the solution of the inequality (12), then the following inequality will be satisfied:

$$
\left\{\begin{aligned}
\mid x(t)- & \int_{0}^{1} G_{\alpha}(t, s) f(s, y(s)) d s-\frac{1}{p^{2}}\left[(p(1-t)+q) \int_{0}^{1} a_{1}(x(s)) d s\right. \\
+ & \left.(q+p t) \int_{0}^{1} a_{2}(x(s)) d s\right] \mid \leq \mathcal{K}_{(\alpha p q)} \epsilon_{\alpha}, \quad t \in \mathcal{J} \\
\mid y(t)- & \int_{0}^{1} G_{\beta}(t, s) g(s, x(s)) d s-\frac{1}{\tilde{p}^{2}}\left[(\tilde{p}(1-t)+\tilde{q}) \int_{0}^{1} \tilde{a}_{1}(y(s)) d s\right. \\
\left.+(\tilde{q}+\tilde{p} t) \int_{0}^{1} \tilde{a}_{2}(y(s)) d s\right] \mid & \leq \mathcal{K}_{(\beta \tilde{p} \tilde{q})} \epsilon_{\beta}, t \in \mathcal{J} .
\end{aligned}\right.
$$

Proof. By Remark 1 (R2), we have

$$
\left\{\begin{array}{l}
{ }^{c} D^{\alpha} x(t)=f(t, y(t))+\phi(t), \quad t \in \mathcal{J}, \\
{ }^{c} D^{\beta} y(t)=g(t, x(t))+\psi(t), \quad t \in \mathcal{J}, \\
p x(0)+q x^{\prime}(0)=\int_{0}^{1} a_{1}(x(s)) d s, \quad p x(1)+q x^{\prime}(1)=\int_{0}^{1} a_{2}(x(s)) d s \\
\tilde{p} y(0)+\tilde{q} y^{\prime}(0)=\int_{0}^{1} \tilde{a}_{1}(y(s)) d s, \quad \tilde{p} y(1)+\tilde{q} y^{\prime}(1)=\int_{0}^{1} \tilde{a}_{2}(y(s)) d s
\end{array}\right.
$$

By Applying Lemma 3, the solution of (15) will be as follows:

$$
\left\{\begin{aligned}
x(t)= & \int_{0}^{1} G_{\alpha}(t, s) f(s, y(s)) d s+\int_{0}^{1} G_{\alpha}(t, s) \phi(s) d s+\frac{1}{p^{2}}\left[(p(1-t)+q) \int_{0}^{1} a_{1}(x(s)) d s\right. \\
& \left.+(q+p t) \int_{0}^{1} a_{2}(x(s)) d s\right], t \in \mathcal{J}, \\
y(t)= & \int_{0}^{1} G_{\beta}(t, s) g(s, x(s)) d s+\int_{0}^{1} G_{\alpha}(t, s) \psi(s) d s+\frac{1}{\tilde{p}^{2}}\left[(\tilde{p}(1-t)+\tilde{q}) \int_{0}^{1} \tilde{a}_{1}(y(s)) d s\right. \\
& \left.+(\tilde{q}+\tilde{p} t) \int_{0}^{1} \tilde{a}_{2}(y(s)) d s\right], t \in \mathcal{J} .
\end{aligned}\right.
$$

Considering first equation of the system (16), we have 


$$
\begin{aligned}
\mid x(t)-\int_{0}^{1} G_{\alpha}(t, s) f(s, y(s)) d s-\frac{1}{p^{2}}\left[(p(1-t)+q) \int_{0}^{1} a_{1}(x(s)) d s\right. & \\
\left.+(q+p t) \int_{0}^{1} a_{2}(x(s)) d s\right] \mid & \leq\left|\int_{0}^{1} G_{\alpha}(t, s) \phi(s) d s\right| \\
& \leq \int_{0}^{1}\left|G_{\alpha}(t, s)\right||\phi(s)| d s .
\end{aligned}
$$

By Remark 1 (R1) and using condition of (A6), we get

$$
\begin{array}{r}
\mid x(t)-\int_{0}^{1} G_{\alpha}(t, s) f(s, y(s)) d s-\frac{1}{p^{2}}\left[(p(1-t)+q) \int_{0}^{1} a_{1}(x(s)) d s\right. \\
\left.+(q+p t) \int_{0}^{1} a_{2}(x(s)) d s\right] \mid \leq \mathcal{K}_{(\alpha p q)} \epsilon_{\alpha} .
\end{array}
$$

Repeating the same procedure for second equation of the system (16), we get

$$
\begin{array}{r}
\mid y(t)-\int_{0}^{1} G_{\beta}(t, s) g(s, x(s)) d s-\frac{1}{\tilde{p}^{2}}\left[(\tilde{p}(1-t)+\tilde{q}) \int_{0}^{1} \tilde{a}_{1}(y(s)) d s\right. \\
\left.+(\tilde{q}+\tilde{p} t) \int_{0}^{1} \tilde{a}_{2}(y(s)) d s\right] \mid \leq \mathcal{K}_{(\beta \tilde{p} \tilde{q})} \epsilon_{\beta} .
\end{array}
$$

Theorem 2. If all the assumptions (A4)-(A6) are fulfilled, then the fractional order coupled system (1) is Ulam-Hyers stable and consequently generalized Ulam-Hyers stable provided that

$$
\left(1-K_{p q}\right)\left(1-K_{\tilde{p} \tilde{q}}\right)-K_{\alpha p q} K_{\beta \tilde{p} \tilde{q}} \neq 0
$$

Proof. Let $(x, y) \in \mathcal{B} \times \mathcal{B}$ be the solution of the system $(15)$ and $(\vartheta, \kappa) \in \mathcal{B} \times \mathcal{B}$ be the unique solution to the following considered system:

$$
\begin{cases}{ }^{c} D^{\alpha} \vartheta(t)=f(t, \kappa(t)), \quad t \in \mathcal{J}, \\ { }^{c} D^{\beta} \kappa(t)=g(t, \vartheta(t)), \quad t \in \mathcal{J}, \\ p \vartheta(0)+q \vartheta^{\prime}(0)=\int_{0}^{1} a_{1}(\vartheta(s)) d s, \quad p \vartheta(1)+q \vartheta^{\prime}(1)=\int_{0}^{1} a_{2}(\vartheta(s)) d s \\ \tilde{p} \kappa(0)+\tilde{q} \kappa^{\prime}(0)=\int_{0}^{1} \tilde{a}_{1}(\kappa(s)) d s, \quad \tilde{p} \kappa(1)+\tilde{q} \kappa^{\prime}(1)=\int_{0}^{1} \tilde{a}_{2}(\kappa(s)) d s\end{cases}
$$

Using Lemma 3, the solution of (19)

$$
\left\{\begin{aligned}
\vartheta(t)= & \int_{0}^{1} G_{\alpha}(t, s) f(s, \kappa(s)) d s+\frac{1}{p^{2}}\left[(p(1-t)+q) \int_{0}^{1} a_{1}(\vartheta(s)) d s\right. \\
& \left.+(q+p t) \int_{0}^{1} a_{2}(\vartheta(s)) d s\right], t \in \mathcal{J}, \\
\kappa(t)= & \int_{0}^{1} G_{\beta}(t, s) g(s, \vartheta(s)) d s+\frac{1}{\tilde{p}^{2}}\left[(\tilde{p}(1-t)+\tilde{q}) \int_{0}^{1} \tilde{a}_{1}(\kappa(s)) d s\right. \\
& \left.+(\tilde{q}+\tilde{p} t) \int_{0}^{1} \tilde{a}_{2}(\kappa(s)) d s\right], t \in \mathcal{J} .
\end{aligned}\right.
$$

We have 


$$
\begin{aligned}
|x(t)-\vartheta(t)|= & \mid x(t)-\int_{0}^{1} G_{\alpha}(t, s) f(s, \kappa(s)) d s-\frac{1}{p^{2}}\left[(p(1-t)+q) \int_{0}^{1} a_{1}(\vartheta(s)) d s\right. \\
& \left.+(q+p t) \int_{0}^{1} a_{2}(\vartheta(s)) d s\right] \mid \\
\leq & \mid x(t)-\int_{0}^{1} G_{\alpha}(t, s) f(s, y(s)) d s-\frac{1}{p^{2}}\left[(p(1-t)+q) \int_{0}^{1} a_{1}(x(s)) d s\right. \\
& \left.+(q+p t) \int_{0}^{1} a_{2}(x(s)) d s\right] \mid \\
& +\left|\int_{0}^{1} G_{\alpha}(t, s) f(s, y(s)) d s-\int_{0}^{1} G_{\alpha}(t, s) f(s, \kappa(s)) d s\right| \\
& +\mid \frac{1}{p^{2}}\left[(p(1-t)+q) \int_{0}^{1} a_{1}(x(s)) d s+(q+p t) \int_{0}^{1} a_{2}(x(s)) d s\right] \\
& -\frac{1}{p^{2}}\left[(p(1-t)+q) \int_{0}^{1} a_{1}(\vartheta(s)) d s+(q+p t) \int_{0}^{1} a_{2}(\vartheta(s)) d s\right] \mid \\
\leq & \mathcal{K}_{\alpha p q} \epsilon_{\alpha}+K_{\alpha p q}|y(t)-\kappa(t)|+K_{p q}|x(t)-\vartheta(t)|,
\end{aligned}
$$

where $K_{\alpha p q}=\mathcal{K}_{(\alpha p q)} K_{f}$.

Hence, we get

$$
\left(1-K_{p q}\right)\|x-\vartheta\|_{\mathcal{B}} \leq \mathcal{K}_{(\alpha p q)} \epsilon_{\alpha}+K_{\alpha p q}\|y-\kappa\|_{\mathcal{B}}
$$

Similarly, we have

$$
\left(1-K_{\tilde{p} \tilde{q}}\right)\|y-\kappa\|_{\mathcal{B}} \leq \mathcal{K}_{(\beta \tilde{p} \tilde{q})} \epsilon_{\beta}+K_{\beta \tilde{p} \tilde{q}}\|x-\vartheta\|_{\mathcal{B}}
$$

where $K_{\beta \tilde{p} \tilde{q}}=\mathcal{K}_{(\beta \tilde{p} \tilde{q})} K_{g}$.

From (21) and (22), it can be written as

$$
\left\{\begin{array}{l}
\left(1-K_{p q}\right)\|x-\vartheta\|_{\mathcal{B}}-K_{\alpha p q}\|y-\kappa\|_{\mathcal{B}} \leq \mathcal{K}_{(\alpha p q)} \epsilon_{\alpha} \\
\left(1-K_{\tilde{p} \tilde{q})}\|y-\kappa\|_{\mathcal{B}}-K_{\beta \tilde{p} \tilde{q}}\|x-\vartheta\|_{\mathcal{B}} \leq \mathcal{K}_{(\beta \tilde{p} \tilde{q})} \epsilon_{\beta}\right.
\end{array}\right.
$$

The matrix representation of (23) is as follows

$$
\left(\begin{array}{cc}
\left(1-K_{p q}\right) & -K_{\alpha p q} \\
-K_{\beta \tilde{p} \tilde{q}} & \left(1-K_{\tilde{p} \tilde{q})}\right.
\end{array}\right)\left(\begin{array}{c}
\|x-\vartheta\|_{\mathcal{B}} \\
\|y-\kappa\|_{\mathcal{B}}
\end{array}\right) \leq\left(\begin{array}{c}
\mathcal{K}_{(\alpha p q)} \epsilon_{\alpha} \\
\mathcal{K}_{(\beta \tilde{p} \tilde{q})} \epsilon_{\beta}
\end{array}\right) .
$$

After simplification of the above inequality, we have

$$
\left(\begin{array}{l}
\|x-\vartheta\|_{\mathcal{B}} \\
\|y-\kappa\|_{\mathcal{B}}
\end{array}\right) \leq\left(\begin{array}{cc}
\frac{\left(1-K_{\tilde{p} \tilde{q})}\right.}{\Delta} & \frac{K_{\alpha p q}}{\Delta} \\
\frac{K_{\beta \tilde{p} \tilde{q}}}{\Delta} & \frac{\left(1-K_{p q}\right)}{\Delta}
\end{array}\right)\left(\begin{array}{l}
\mathcal{K}_{(\alpha p q)} \epsilon_{\alpha} \\
\mathcal{K}_{(\beta \tilde{p} \tilde{q})} \epsilon_{\beta}
\end{array}\right)
$$

where $\Delta=\left(1-K_{p q}\right)\left(1-K_{\tilde{p} \tilde{q}}\right)-K_{\alpha p q} K_{\beta \tilde{p} \tilde{q}} \neq 0$.

Further simplification gives

$$
\begin{aligned}
\|x-\vartheta\|_{\mathcal{B}} & \leq \frac{\left(1-K_{\tilde{p} \tilde{q})} \mathcal{K}_{(\alpha p q)} \epsilon_{\alpha}\right.}{\Delta}+\frac{K_{\alpha p q} \mathcal{K}_{(\beta \tilde{p} \tilde{q})} \epsilon_{\beta}}{\Delta} \\
\|y-\kappa\|_{\mathcal{B}} & \leq \frac{K_{\beta \tilde{p} \tilde{q}} \mathcal{K}_{(\alpha p q)} \epsilon_{\alpha}}{\Delta}+\frac{\left(1-K_{p q}\right) \mathcal{K}_{(\beta \tilde{p} \tilde{q})} \epsilon_{\beta}}{\Delta}
\end{aligned}
$$


From inequalities (24) and (25), we have

$$
\begin{aligned}
\|x-\vartheta\|_{\mathcal{B}}+\|y-\kappa\|_{\mathcal{B}} \leq & \frac{\left(1-K_{\tilde{p} \tilde{q})}\right) \mathcal{K}_{(\alpha p q)} \epsilon_{\alpha}}{\Delta}+\frac{K_{\alpha p q} \mathcal{K}_{(\beta \tilde{p} \tilde{q})} \epsilon_{\beta}}{\Delta}+\frac{K_{\beta \tilde{p} \tilde{q}} \mathcal{K}_{(\alpha p q)} \epsilon_{\alpha}}{\Delta} \\
& +\frac{\left(1-K_{p q}\right) \mathcal{K}_{(\beta \tilde{p} \tilde{q})} \epsilon_{\beta}}{\Delta}
\end{aligned}
$$

Therefore, we have

$$
\|(x, y)-(\vartheta, \kappa)\|_{\mathcal{B} \times \mathcal{B}} \leq \mathcal{K}_{(\alpha \beta p q \tilde{p} \tilde{q})} \epsilon,
$$

where $\epsilon=\max \left\{\epsilon_{\alpha}, \epsilon_{\beta}\right\}$ and

$$
\mathcal{K}_{(\alpha \beta p q \tilde{q} \tilde{q})}=\frac{\left(1-K_{\tilde{p} \tilde{q})} \mathcal{K}_{(\alpha p q)}\right.}{\Delta}+\frac{K_{\alpha p q} \mathcal{K}_{(\beta \tilde{p} \tilde{q})}}{\Delta}+\frac{K_{\beta \tilde{p} \tilde{q}} \mathcal{K}_{(\alpha p q)}}{\Delta}+\frac{\left(1-K_{p q}\right) \mathcal{K}_{(\beta \tilde{p} \tilde{q})}}{\Delta} .
$$

Hence, by inequality (26), we can conclude that the coupled system (1) is Ulam-Hyers stable. Further, inequality (26) can be written as

$$
\|(x, y)-(\vartheta, \kappa)\|_{\mathcal{B} \times \mathcal{B}} \leq \Psi(\epsilon), \text { where } \Psi(0)=0 .
$$

By inequality (27), we further conclude that the coupled system (1) is generalized Ulam-Hyers stable.

\section{Application}

Example 1. We consider the following fractional order coupled system:

$$
\left\{\begin{array}{l}
{ }^{c} D^{\alpha} x(t)=\frac{1}{(t+7)^{2}} \frac{|y(t)|}{1+|y(t)|}, \alpha \in(1,2], t \in \mathcal{J}=[0,1] \\
{ }^{c} D^{\beta} y(t)=\frac{1}{100}[t \cos x(t)+x(t) \sin t], \beta \in(1,2], t \in \mathcal{J}=[0,1] \\
x(0)+x^{\prime}(0)=\int_{0}^{1} \frac{|x(s)|}{13+|x(s)|} d s, \quad x(1)+x^{\prime}(1)=\int_{0}^{1} \frac{|x(s)|}{15+|x(s)|} d s \\
y(0)+y^{\prime}(0)=\int_{0}^{1} \frac{1}{26}[\cos y(s)+\sin y(s)] d s, \quad y(1)+y^{\prime}(1)=\int_{0}^{1} \frac{1}{30}[\cos y(s)+\sin y(s)] d s
\end{array}\right.
$$

By comparing the coupled systems (28) to (1), the following values are derived:

$$
p=q=\tilde{p}=\tilde{q}=1, K_{a_{1}}=K_{\tilde{a}_{1}}=\frac{1}{13} \text { and } K_{a_{2}}=K_{\tilde{a}_{2}}=\frac{1}{15} .
$$

Here,

$$
f(t, y(t))=\frac{1}{(t+7)^{2}} \frac{|y(t)|}{1+|y(t)|} \text { and } g(t, x(t))=\frac{1}{100}[t \cos x(t)+x(t) \sin t] .
$$

As, $|f(t, y)-f(t, \tilde{y})| \leq \frac{1}{49}|y-\tilde{y}|$ and $|g(t, x)-g(t, \tilde{x})| \leq \frac{1}{50}|x-\tilde{x}|$, therefore (A4) is satisfied with $K_{f}=\frac{1}{49}$ and $K_{g}=\frac{1}{50}$. Further, we have

$$
\begin{aligned}
\phi_{1} & =\left(\frac{1}{\Gamma(\beta+1)}+\frac{2(\tilde{p}+\tilde{q})}{\Gamma(\beta+1)}+\frac{2\left(\tilde{q}^{2}+\tilde{p} \tilde{q}\right)}{\tilde{p}^{2} \Gamma(\beta)}\right) K_{g}+\frac{(p+q)\left(K_{a_{1}}+K_{a_{2}}\right)}{p^{2}} \\
& =\left(\frac{1}{\Gamma(\beta+1)}+\frac{4}{\Gamma(\beta+1)}+\frac{4}{\Gamma(\beta)}\right) \frac{1}{50}+2\left(\frac{1}{13}+\frac{1}{15}\right) \\
& <1 .
\end{aligned}
$$


Similarly, $\phi_{2}<1$. Hence, the coupled system (28) has a unique solution. Moreover, the condition $\left(1-K_{p q}\right)\left(1-K_{\tilde{p} \tilde{q}}\right)-K_{\alpha p q} K_{\beta \tilde{p} \tilde{q}} \neq 0$ in Theorem 2 is also satisfied. Therefore, coupled system (28) is Ulam-Hyers stable as well as generalized Ulam-Hyers stable.

\section{Conclusions}

Here we have studied the existence and uniqueness of the solutions as well as the stability for a coupled system of fractional order $\alpha \in(1,2]$ differential equation with integral boundary conditions. We have discussed two types of stability, called Ulam-Hyers stability and generalized Ulam-Hyers stability. As a future work, one can generalize the same concept of stability to a neutral time delay system/inclusion as well as state delay system/inclusion (finite and infinite delay), which have some useful scientific applications. This will enhance a new direction of research: a special kind of phase space to be used for the study of controllability and stability of an infinite delay system/inclusion.

Author Contributions: Both the authors have contributed equally in the article.

Acknowledgments: We authors are thankful to referees for their valuable suggestions and comments which greatly improved the manuscript.

Conflicts of Interest: The authors declare no conflicts of interest.

\section{References}

1. Muslim, M.; Kumar, A. Controllability of fractional differential equation of order $\alpha \in(1,2]$ with non-instantaneous impulses. Asian J. Control 2017. [CrossRef]

2. Yang, Q.; Chen, D. Fractional calculus in image processing: A review. Fract. Calcul. Appl. Anal. 2016, 19, 1222-1249. [CrossRef]

3. Magin, R.L. Fractional Calculus in Bioengineering; Begell House: Redding, CA, USA, 2006.

4. Kilbas, A.A.; Srivastava, H.M.; Trujillo, J.J. Theory and Applications of Fractional Differential Equations; North-Holland Mathematics Studies; Elsevier Science Inc.: New York, NY, USA, 2006; Volume 204.

5. Miller, K.S.; Ross, B. An Introduction to Fractional Calculus and Fractional Differential Equations; A Wiley Interscience Publication, Wiley: New York, NY, USA, 1993.

6. Hilfer, R. Applications of Fractional Calculus in Physics; World Scientific: Singapore, 2000.

7. Khan, A.; Shah, K.; Li, Y.; Khan, T.S. Ulam type stability for a coupled system of boundary value problems of nonlinear fractional differential. Equ. J. Funct. Spaces 2017, 2, 1-8. [CrossRef]

8. Ahmad, B.; Nieto, J.J. Existence results for nonlinear boundary value problems of fractional integro-differential equations with integral boundary conditions. Bound. Value Probl. 2009, 2009. [CrossRef]

9. Muslim, M.; Kumar, A.; Agarwal, R.P. Exact controllability of fractional integro-differential systems of order $\alpha \in(1,2]$ with deviated argument. Analele Universitătii Oradea 2017, XXIV, 185-194.

10. Chalishajar, D.N.; Karthikeyan, K. Boundary value Problems for Impulsive Fractional Evolution Integrodifferential Equations with Gronwall's inequality in Banach spaces. J. Discontinuity Nonlinearity Complex. 2014, 3, 33-48. [CrossRef]

11. Chalishajar, D.N.; Karthikeyan, K. Existence and Uniqueness Results for Boundary Value Problems of Higher order Fractional Integro-differential Equations Involving Gronwall's Inequality in Banach spaces. Acta Math. Sci. Ser. A 2013, 33B, 758-772. [CrossRef]

12. Ahmad, B.; Alsaedi, A.; Alghamdi, B.S. Analytic approximation of solutions of the forced Duffing equation with integral boundary conditions. Nonlinear Anal. Real World Appl. 2008, 9, 1727-1740. [CrossRef]

13. Ulam, S.M. A Collection of the Mathematical Problems; Interscience: New York, NY, USA, 1960.

14. Hyers, D.H. On the stability of the linear functional equation. Proc. Natl. Acad. Sci. USA 1941, 27, 222-224. [CrossRef] [PubMed]

15. Jung, S.M. Hyers-Ulam stability of linear differential equations of first order. Appl. Math. Lett. 2006, 19, 854-858. [CrossRef]

16. Wang, J.; Feckan, M. A general class of impulsive evolution equations. Topol. Methods Nonlinear Anal. 2015, 46, 915-933. [CrossRef] 
17. Muslim, M.; Kumar, A.; Fečkan, M. Existence, uniqueness and stability of solutions to second order nonlinear differential equations with non-instantaneous impulses. J. King Saud Univ. Sci. 2016. [CrossRef]

18. Rus, I.A. Ulam stabilities of ordinary differential equations in a Banach space. Carpath. J. Math. 2010, 26, 103-107.

(C) 2018 by the authors. Licensee MDPI, Basel, Switzerland. This article is an open access article distributed under the terms and conditions of the Creative Commons Attribution (CC BY) license (http://creativecommons.org/licenses/by/4.0/). 